\title{
CALL FOR ABSTRACTS/DEMANDE DE RÉSUMÉS
}

\section{3rd Meeting of the Canadian Congress of Neurological Sciences June 16-20, 1998}

\section{CALL FOR ABSTRACTS \\ Scientific Papers and Posters}

1. Abstracts for the scientific program must be submitted on the official 1998 Congress Abstract Form.

2. Work presented must conform with MRC guidelines for experimental procedures.

3. Scientific material presented at this meeting should not have been published or presented at other national or international meetings.

4. It is suggested that presentations emphasize the significance of the results and general principles involved rather than ordinary methods and procedures.

\section{ABSTRACTS MUST BE RECEIVED BY} JANUARY 15, 1998.

6. Abstracts accepted for presentation will be published in the Canadian Journal of Neurological Sciences.

\section{3e Assemblée annuelle du Congrès canadien des sciences neurologiques 16 - 20 juin 1998}

\section{DEMANDE DE RÉSUMÉS \\ Présentations orales et par affiches des résumés}

1. Les résumés pour le programme scientifique doivent être soumis sur le formulaire officiel du congrès désigné à cet effet.

2. Le travail présenté dans les résumés doit être conforme aux principes du C.R.M. relatifs aux procédures expérimentales.

3. Le matériel scientifique présenté à ce congrès ne doit pas avoir été publié ou présenté à d'autres congrès nationaux ou internationaux.

4. Il est recommandé de mettre l'emphase sur les résultats et les principes généraux plutôt que d'élaborer sur la méthodologie.

\section{LES RÉSUMÉS DOIVENT ÊTRE REÇUS AVANT LE 15 JANVIER 1998.}

6. Les résumés acceptés paraîtront dans le Journal canadien des sciences neurologiques.

For further information, please contact / Pour de plus amples renseignements, veuillez contacter :

Canadian Congress of Neurological Sciences/Congrès canadien des sciences neurologiques

Suite 810, 906 - 12 Avenue SW

Calgary, Alberta, Canada T2R 1K7

Tel / Tél. : (403) 229-9544 Fax: (403) 229-1661 Email / Courrier électronique : brains@ccns.org 